\title{
Trends in Endodontic Protocols Amongst Dentists in Lahore
}

\author{
Saood Khan Lodhi ${ }^{1}$ \\ Saroosh Ehsan ${ }^{2}$ \\ Adeela Rafique $^{3}$ \\ Muhammad Nasir Saleem ${ }^{4}$
}

BDS

\author{
BDS, FCPS \\ BDS, MSc \\ BDS, FCPS, MSc
}

OBJECTIVES: To determine the trends in endodontic protocols amongst practicing dentists in teaching institutions and private clinics in Lahore.

METHODOLOGY: A cross sectional survey was conducted amongst dentists working in the teaching institutions and in the private clinics of Lahore. A pre-piloted questionnaire survey adopted from Kohli et al with slight modifications was used. In this descriptive study 270 practicing dentists were selected using consecutive sampling with a $5 \%$ level of significance and margin of error.

RESULTS: The 270 respondents had a mean age of $28.16 \pm 6.18$ participated in this study among which $111(41.1 \%)$ were males and $159(58.9 \%)$ were females with the mean age of $30.28 \pm 7.416$ and $26.68 \pm 4.62$ respectively (12.2\%) were consultants, $175(64.8 \%)$ were general dentists whereas $62(23 \%)$ were postgraduate residents.

In our study population, 76(28.1\%) dentists were using magnification among which 67(88.2\%) were using loupes and 9(11.8\%) were using microscope. The prevalence of the rubber dam usage for isolation among our respondents was $110(40.7 \%)$ only, 201(74.4\%) dentists reported for routine removal of smear layer.

Manual canal preparation technique was the most commonly used method practiced by 149(55.2\%) dentists. 136(50.4\%) dentists were using beveled needles for irrigation, whereas only 71(26.3\%) were using side venting irrigation needles. Majority of the dentists (45.2\%) were not using any method to increase the efficacy of the irrigants.

CONCLUSION: There are deficiencies in the endodontic protocols followed by majority of the dentists in Lahore and these short comings should be addressed by governing bodies.

KEYWORDS: Root canal therapy, Endodontic, Practice Patterns, Dentists

HOW TO CITE: Lodhi Sk, Ehsan S, Rafique A, Saleem MN. Trends in endodontic protocols amongst dentists in Lahore. J Pak Dent Assoc 2020;29(4):239-245.

DOI: https://doi.org/10.25301/JPDA.294.239

Received: 30 July 2020, Accepted: 08 September 2020

\section{INTRODUCTION}

$\mathrm{E}$ ndodontics has evolved at a very fast pace in the last two decades with the help of both technology and a much-improved understanding of scientific concepts. Under the umbrella of evidence-based practices, endodontics has propelled from blind preparation of canals to endodontics under the microscope with effective irrigation and disinfection procedures. The dental professionals are now faced with a plethora of choices in terms of equipment

1. Postgraduate Resident, Department of Operative Dentistry, FMH College of Medicine \& Dentistry, Lahore, Pakistan.

2. Associate Professor, Department of Operative Dentistry, FMH College of Medicine \& Dentistry, Lahore, Pakistan.

3. Associate Professor, Department of Operative Dentistry, FMH College of Medicine \& Dentistry, Lahore, Pakistan.

4. Professor, Department of Operative Dentistry, CMH Lahore Medical \& Dental College, Lahore, Pakistan

Corresponding author: “Dr. Saood Khan Lodhi" < ssaoodkhan496@gmail.com > selection, material selection and applied technology. ${ }^{1}$ The outcome of root canal treatment is greatly reliant on maintenance of treatment standards. ${ }^{2}$ However, several studies in different parts of the world, have reported the majority of dentists not fulfilling the standard guidelines. ${ }^{3-7}$ It is therefore important to carry out a survey among dentists of a given region to see their implementation of evidence based practice in their endodontic practices. ${ }^{1}$ Studies conducted from university clinics and specialists have reported high success rate of endodontic treatment of more than $90 \%$, However, these statistics do not define the exact success rate of endodontic treatment in general dental practice. The success rate of endodontic treatment in general dental practice estimates to $65-75 \%$ only. ${ }^{9}$ The inconsistency in success rate noted may indicate a difference in the technical quality of endodontic treatment being performed by dentists with different experience. Considering our patients' health, it would be of utmost importance to ascertain the practices 
adopted by dental practitioners of a given region. Various studies conducted in different parts of the world have shown that despite the number of new innovations in dentistry, deficiencies are seen in endodontic treatment provided by practicing dentists. A national survey on trends in contemporary Endodontic practice in Pakistan done by Bhatti et al showed deficiencies among dental practitioners providing endodontic treatment. ${ }^{1}$ The rationale of this study was to collect data regarding knowledge, materials, methods, and attitudes exercised during endodontic treatment by dentists in Lahore to assess their practices and to help in enhancing the quality of endodontic treatment. No such study has been conducted at a regional level in Lahore.

\section{METHODOLOGY}

A cross sectional survey was conducted amongst dentists working in teaching institutions and in private clinics of Lahore. After approval from the Institutional Review Board (IRB) of Fatima Memorial Hospital (FMH-03-2019-IRB586-M), the study was initiated and data collection was completed in 3 months. A sample size of 278 dentists was selected using $5 \%$ level of significance, $95 \%$ confidence interval and 5\% margin of error. Dentists practicing in private practices as well as in teaching institutes of Lahore were included in the inclusion criteria whereas undergraduate dental students and dental hygienists were excluded from the study. A pre piloted questionnaire survey adopted from Kohli et al with slight modifications was used. ${ }^{10}$ The selfadministered questionnaire comprising of 26 questions covered various aspects of endodontic treatment including personal and demographic detail. The questionnaire was filled by general dentists, post-graduate residents and consultants. The anonymity of the respondents was maintained. The survey included questions concerning various aspects of endodontic treatment protocol including use of rubber dam, techniques for working length determination, use of magnification, technique for root canal instrumentation, choice of irrigants with adjuncts and needle choice, choice of intracanal medicaments, obturation techniques and choice of material for sealer. The responses were analyzed in SPSS VERSION 21 to calculate frequencies and percentages.

\section{RESULTS}

A total of 270 respondents with mean age of $28.16 \pm 6.179$ participated in this study among which $111(41.1 \%)$ were males and 159(58.9\%) were females with the mean age of $30.22 \pm 7.416$ and $26.88 \pm 4.620$ respectively. Distribution of the respondents with respect to their specialties can be seen in Table 1. Among our respondents, $120(44.4 \%)$ were in academic practice, $65(24.1 \%)$ were in private practice and remaining were practicing both i-e. private and academic. (Table:1)

The use of the magnification in our study population was $76(28.1 \%)$, among which $67(24.8 \%)$ were using loupes and only $9(3.3 \%)$ of the respondents were using dental

Table 1: Demographics

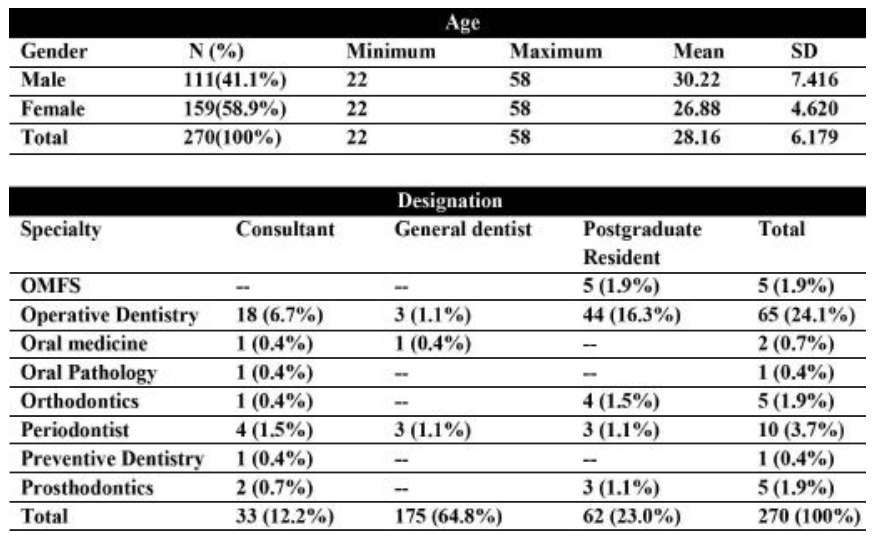

176 respondents did not mention their specialty.

(--) means no respondent

\begin{tabular}{lll}
\hline Type of practice & Frequency & Percentage \\
\hline Academic & 120 & 44.4 \\
\hline Private & 65 & 24.1 \\
\hline Both & 85 & 31.5 \\
\hline Total & 270 & 100 \\
\hline
\end{tabular}

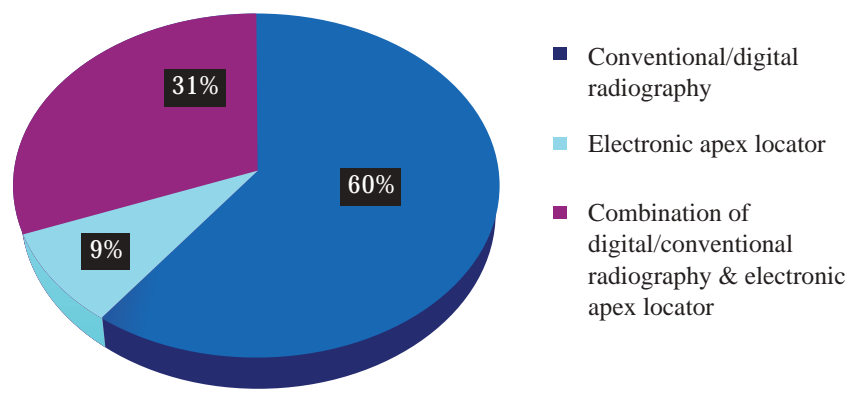

Preferred method of working length determination

Figure 1

operating microscope. The prevalence of the rubber dam usage for isolation by the dentists was 110(40.7\%). (Table:2)

Among our study population the use of the Combination of digital/conventional radiography and electronic apex locator as a method of working length determination was $83(31.1 \%)$ and the use of Conventional/Digital radiography only was 161(59.6\%). (Figure:1)

Manual canal preparation technique was the most commonly used method of canal preparation practiced by $149(55.2 \%)$ followed by combination of manual and engine driven instruments by 66(24.4\%), whereas only 41(15.2\%) were using engine driven instruments as a preferred method 
Table 2: Frequency of use of Magnification and Rubber dam

\begin{tabular}{lll} 
Do you use Magnification? & Frequency & Percentage \\
\hline Yes & 76 & 28.1 \\
\hline No & 194 & 71.9 \\
\hline Total & 270 & 100 \\
\hline If Yes & & \\
\hline Loupes & 67 & 24.8 \\
\hline Microscope & 9 & 3.3 \\
\hline Total & 76 & 28.1 \\
\hline Do you use rubber dam? & & \\
\hline Yes & 110 & 40.7 \\
\hline No & 160 & 59.3 \\
\hline Total & 270 & 100 \\
\hline
\end{tabular}

Table 3: Canal Preparation technique and method

\begin{tabular}{|c|c|c|}
\hline Question & Frequency & Percentage \\
\hline \multicolumn{3}{|c|}{ Which technique of canal preparation do you use in routine cases? } \\
\hline Engine driven preparation & 41 & 15.2 \\
\hline Manual preparation & 149 & 55.2 \\
\hline Combination & 66 & 24.4 \\
\hline Total & 256 & 94.8 \\
\hline \multicolumn{3}{|c|}{ Which method of canal preparation do you use in routine cases? } \\
\hline Step-back & 127 & 47.0 \\
\hline Crown down & 71 & 26.3 \\
\hline Combination & 58 & 21.5 \\
\hline Total & 256 & 94.8 \\
\hline
\end{tabular}

of root canal preparation. Step-back technique was the most frequently used method of canal preparation used by $127(47 \%)$ of the dentists. (Table:3)

The most commonly used engine driven instrument for preparation of root canal system was Protaper universal, preferred by $140(51.9 \%)$ of the dentists. (Figure:2)

Among our study population, majority i.e. 201(74.4\%) dentists reported for routine removal of smear layer. For smear layer removal the use of Sodium Hypochlorite primarily after access opening was $68.5 \%$ with a mean concentration of $2.75 \pm 1.70$. Almost fifty percent i.e.50.4\%

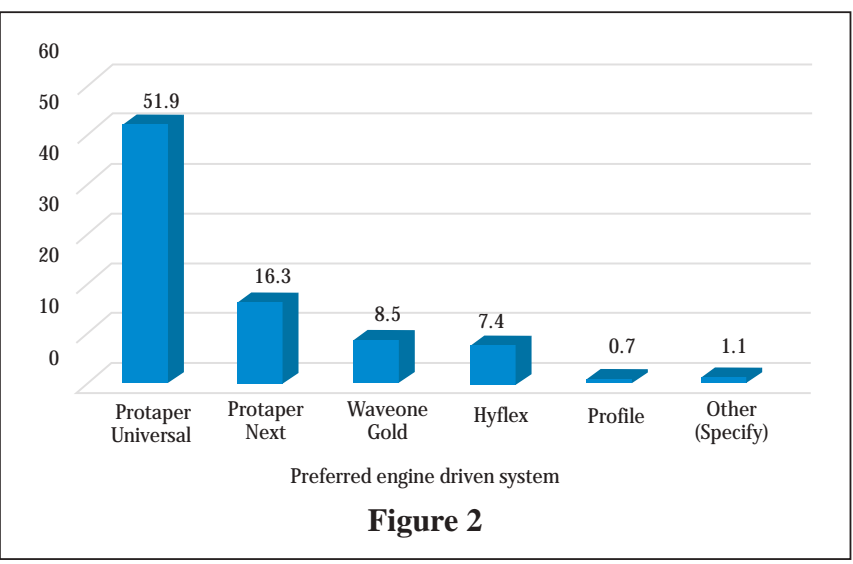

of the dentists were using beveled needles for intracanal irrigation followed by side venting irrigation needles which were used by $26.3 \%$ of the respondents. Almost $50 \%$ of the respondents did not have any idea about the gauge of needle used for intracanal irrigation. When it was asked about the
Table 4: Cleaning of the Root Canal System

\begin{tabular}{lll}
\hline & & \\
\hline Do you routinely remove smear layer? & 201 & 74.4 \\
\hline Yes & 45 & 16.7 \\
\hline No & 246 & 91.1 \\
\hline Total & 185 & 68.5 \\
\hline Which irrigants do you use primarily after access opening? & 30 & 11.1 \\
\hline Sodium Hypochlorite & 28 & 10.4 \\
\hline EDTA & 10 & 3.7 \\
\hline Normal Saline & 4 & 1.5 \\
\hline Chlorhexidine & 3 & 1.1 \\
\hline MTAD & 8 & 3 \\
\hline Sterile water & 268 & 99.3 \\
\hline Combination & $2.75 \pm 1.70$ & \\
\hline Total & Frequency & Percentage \\
\hline What concentration of sodium hypochlorite do you use primarily? & 71 & 26.3 \\
\hline Which needle do you use for intracanal irrigation? & 48 & 17.8 \\
\hline Side venting irrigation needles & 12 & 4.4 \\
\hline Flat ended needles & 136 & 50.4 \\
\hline Multi-vented needles & 267 & 98.9 \\
\hline Beveled needles & & \\
\hline Total & 103 & 38.1 \\
\hline Which gauge of the needle do you use for intracanal irrigation? & 28 & 10.4 \\
\hline $24 G$ & 15 & 5.6 \\
\hline 27G & 146 & 54.1 \\
\hline 30G & 124 & 45.9 \\
\hline Total & 16 & \\
\hline Missing Response & 33 & 5.9 \\
\hline Do you use any adjuvants to increase the efficacy of the irrigants? & 20 & 7.2 \\
\hline Sonic activation & 9 & 3.3 \\
\hline Ultrasonic activation & 66 & 24.4 \\
\hline Subsonic activator (Endoactivator) & 122 & 45.2 \\
\hline Negative Pressure devices & 266 & 98.5 \\
\hline Manual activation & & \\
\hline I do not use any & & \\
\hline Total & & \\
\hline & & \\
\hline
\end{tabular}

Table 5: Intracanal medicament and sealers

\begin{tabular}{lll}
$\begin{array}{l}\text { Which intracanal medicament do you use after irrigation } \\
\text { for inter appointment disinfection? }\end{array}$ & Frequency & Percentage \\
\hline Calcium hydroxide & 203 & 75.2 \\
\hline Ledermix & 41 & 15.2 \\
\hline Triple antibiotic paste & 15 & 5.6 \\
\hline I do not place any & 3 & 1.1 \\
\hline Other (Specify) & 8 & 3.0 \\
\hline Total & 270 & 100 \\
\hline Which sealer do you? & & \\
\hline Zinc oxide Eugenol based & 89 & 33 \\
\hline Ca(OH) based & 141 & 52.2 \\
Resin based & 22 & 8.1 \\
\hline Glass ionomer based & 11 & 4.1 \\
\hline Total & 263 & 97.4
\end{tabular}

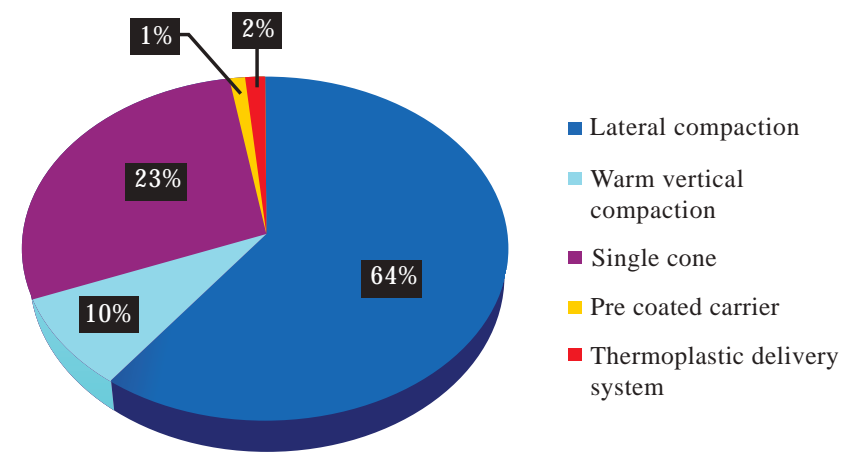

Preferred method of obturation used by dentists

Figure 3

use of any adjuvants to increase the efficacy of the irrigants it was found that majority $(45.2 \%)$ were not using any method to increase the efficacy of the irrigants whereas only $24.4 \%$ of the respondents reported to use manual 
activation method of irrigant activation. (Table:4)

Two third of the practicing dentists i.e. 203(75.2\%) were using calcium hydroxide as an intracanal medicament for achieving inter-appointment disinfection. Most commonly used root canal sealer was calcium hydroxide (52\%) followed by Zinc Oxide Eugenol based sealers (33\%). (Table:5)

Lateral compaction $(64.1 \%)$ was the preferred method of obturation. (Figure:3)

\section{DISCUSSION}

This study evaluated the trends in endodontic protocols amongst practicing dentists in Lahore. A sample from academic institutes and private practices was selected. The distribution of male to female dentists were $41.1 \%$ and $58.9 \%$ which is in accordance with the fact that the number of female dental surgeons is higher than males in Pakistan. ${ }^{11}$

The mean age of male to female dentists were $30.22 \pm 7.416$ and $26.88 \pm 4.620$ respectively. The majority of the respondents were below 30 years of age with their work experience of less than 10 years. With the establishment of many new private dental colleges in Pakistan a very large number of young practitioners are present. ${ }^{12}$

The use of rubber dam for isolation is integral in reducing microbial contamination, it decreases risks such as swallowing of instruments and medicaments, and thus increases the overall success rate of the endodontic treatment. In this study, the percentage of dentists who used rubber dam routinely for isolation was $40.7 \%$, which is similar to other surveys performed in different countries by Palmer $30.3 \%$, Unal 5.1\%, Bhatti $39.6 \%$ and Raoof $16 \% .^{1,2,13,14}$ Considering the current circumstances where COVID-19 pandemic has adversely affected the dentistry services, the use of rubber dam is very important for carrying out aerosol generating procedures in the dental office to reduce the risk of cross contamination. ${ }^{15}$

Over the past few decades due to technological innovations in dentistry the benefits of the use of magnification during endodontic treatment cannot be ignored as it helps in location of missed canals, calcified canals and management of iatrogenic complications. ${ }^{16}$ Despite the proven advantages, in this study, $72 \%$ of the dentists did not use any means of magnification, $24.8 \%$ of the dentists were using loupes and only $3.3 \%$ were using endodontic microscope. The results are similar to a study done by Bhatti et al in which $78.3 \%$ of the practitioners do not use any magnification during root canal treatment. ${ }^{1}$

Determination of working length is a critical step during biomechanical preparation of root canals as inaccuracies during this stage can lead to treatment failure. ${ }^{17}$ The exact location of apical constriction is impossible to determine with conventional or digital radiographs alone because of its variation i.e. 1-3 $\mathrm{mm}$ from the radiographic apex. Electronic apex locators are a major paradigm shift in endodontics as they help in precise location of apical constriction. ${ }^{18}$ In this study, $59.6 \%$ of the dentists were using conventional/ digital radiographs alone whereas only $31.1 \%$ of the practicing dentists were using the Combination of digital/conventional radiography and electronic apex locator for determining of working length. These results are similar to studies conducted in Turkey, Iran, UK, USA by Unal et al, Raoof et al, Palmer et al, Lee at el where $77 \%, 71.3 \%, 57.3 \%, 52.5 \%$ were using conventional/digital radiographs alone whereas the percentage of dentists using the combination of digital/conventional radiography and electronic apex locator were $12.8 \%, 35.7 \%, 34.5 \%$, $8.9 \% .^{1,13,14,19}$

Root canal preparation is one of the key steps which has a major influence on the outcome of endodontic treatment. Various canal preparation techniques are used during cleaning and shaping of the root canal system. ${ }^{20}$ One of the technological advances in endodontics is the introduction of engine driven file systems, which provides a well tapered canal with decreased procedural errors as compared to conventional root canal preparation techniques with stainless steel hand instruments. ${ }^{21}$ Although the use of different engine driven file systems in irregular canals or canals with morphological variations is still a matter of debate, however, they can be used in majority of the cases. ${ }^{20}$ Despite the proven advantages of engine driven systems, in the present study, most practicing dentists still used manual canal preparation with hand instruments as the preferred method of root canal preparation.

One of the findings of this study is that although $40 \%$ of our respondents used rubber dam in contrast to $15.2 \%$ pursuing engine driven systems for canal preparation. A possible explanation of this variation can be that most of the graduates were young dentists who recently graduated and were under the direct and indirect supervision of their respective supervisors in teaching institutions where use of rubber dam is mandatory to follow the proper protocol of endodontics. However, because of inexperience the use of engine driven instruments for root canal preparation is limited.

Apical extrusion of debris and irrigant solutions is one of major causes of painful flare-ups which can affect the quality of life of the patients. Canal preparation method can play an important role in avoiding such unforeseen conditions. Various studies conducted in different parts of the world have shown crown down technique to be superior than step-back technique in terms of less generation of 
stresses along the files, decreased extrusion of apical debris and irrigants. ${ }^{22}$ Nonetheless, almost half i.e. $47 \%$ of the dentists in our study were more inclined towards using stepback technique as a preferred method of canal preparation.

Different type of engine driven instruments are available based on the design characteristics, rotating motion, torque and speed of use. ${ }^{21}$ The rotating motions used in these systems are rotation and reciprocation. ${ }^{23}$ After the advent of these systems, the use of continuous rotating motion became popular, however contemporary methods of root canal preparation involve reciprocation motion with a more user-friendly single file system. ${ }^{21,23}$ Regardless of the advantages of reciprocating systems, most commonly used system in our setting was Protaper Universal, which is a multi-file progressive taper rotary system.

During mechanical instrumentation of the root canal system, a layer of hard tissue debris known as smear layer is deposited along the root canal walls which prevents irrigants and intracanal medicaments to get rid of the microorganisms. There is consensus on this topic that complete chemo mechanical debridement including removal of smear layer is one of the major goals of endodontic treatment. ${ }^{24}$ Intracanal irrigation with $0.5-5.25 \%$ sodium hypochlorite is considered a gold standard antimicrobial agent to dissolve organic component of smear layer. ${ }^{25}$ In this study the use of sodium hypochlorite primarily after access opening was practiced by $68.5 \%$ of the dentists. The results are consistent with the findings of studies done by Palmer et al in UK who reported $75 \%$ and Unal et al in Turkey showed $73 \%$ of the dentists practiced use of sodium hypochlorite primarily after access opening. ${ }^{13,14}$

In contrast to this, a study conducted in Iran by Raoof et al showed $42.9 \%$ of the dentists using sodium hypochlorite whereas as $61.8 \%$ using normal saline for irrigation. ${ }^{2}$ These findings are further supported by studies done by Bhatti et al in Pakistan who reported 39.1\% whereas Gaikwad et al in India showed only $12.5 \%$ of the dentists using sodium hypochlorite and $53 \%$ using combination of sodium hypochlorite and normal saline. ${ }^{1,26}$

The aim to achieve 3-dimensional cleaning of the root canal space is not possible without irrigation solution reaching the apical one third of the working length followed by activation of irrigant with different activation methods as areas like isthmuses, apical deltas etc are not negotiable even with modern canal preparation methods. ${ }^{25}$ In this survey $45.2 \%$ of the dentists were not using any irrigant activation method to increase the efficacy of irrigant whereas only $24.4 \%$ used manual method of irrigant activation. With growing evidence, it has become evident that irrigant activation with ultrasonics, sonic activation, or negative pressure devices is very important for irrigant penetration in to the apical one third of the canal for achieving maximum disinfection of the canal..$^{25,27}$

Direct irrigation of the canal with positive pressure irrigation needles is one of the most commonly used irrigation methods taught in undergraduate schools. For routine endodontic procedures a $27 \mathrm{G}$ needle placed $3 \mathrm{~mm}$ from the apex of a canal prepared is sufficient. ${ }^{24} 45.9 \%$ of the respondents showed missing response when they were asked about the needle gauge representing the practicing dentists have lack of knowledge making the answer of this question invalid. New studies should be conducted to find answer to this question.

Calcium hydroxide is considered as a gold standard intracanal medicament to achieve and maintain interappointment disinfection. ${ }^{28}$ In this study $75.2 \%$ of the respondents used Calcium hydroxide as intracanal medicament. This is consistent with the findings of other studies by Bhatti et al 67.2\%, Unal et al 53\%, Palmer et al $60 \% .^{1,13,14}$ In contrast a study by Iqbal et al showed that 55 $\%$ of the dentists using formocresol whereas only $5 \%$ using calcium hydroxide as intracanal medicament. ${ }^{29}$

The main purpose of root canal obturation is to provide three dimensional corono-apical seal of the root canal system to impede the traffic of saliva, bacteria and their virulence and other irritating factors which could lead to post treatment disease. Various methods of root canal obturation are being used for this. ${ }^{30}$

In this study the most common method of obturation was lateral condensation done by $64.1 \%$ of the dentists followed by single cone obturation done by $23 \%$ of the dentists. The results are consistent with the studies done by Gaikwind et al and Azhar et al in which $78.2 \%$ and $81 \%$ of the practicing dentists were using lateral condensation as a method to achieve three dimensional obturation of the root canal system. ${ }^{26,29}$

In this study most commonly used root canal sealer was calcium hydroxide $(52 \%)$ followed by Zinc Oxide Eugenol based sealers $(33 \%)$. These findings are contradictory with the findings of studies done by Lee et al in USA in which Zinc oxide eugenol sealer was used by $74.6 \%$ of the dentists. ${ }^{19}$ Study done by Palmer et al in UK also showed that $56 \%$ of the dentists using Zinc oxide eugenol as a root canal sealer. ${ }^{13}$

\section{CONCLUSION}

This study has shown that there are many shortcomings and gaps in the endodontic protocols followed by most of the dentists in Lahore. This study highlights the need for updating their knowledge and practices. This can eventually become a basis to suggest a standardized treatment guideline. 


\section{LIMITATIONS}

Most of the sample size consisted of young dentists who are at the start of their career and may be cannot afford the use of expensive gadgets i.e. Magnification devices; apex locators; digital periapical radiographs and rotary instrumentation etc.

\section{RECOMMENDATIONS}

These shortcomings should be addressed by the governing dental bodies and faculties at the institutional level and continuing dental education modules should be made compulsory for all general practitioners. Provision of handson courses by the endodontists can be of greater value in this technically challenging field.

\section{ACKNOWLEDGEMENT}

The authors wish to acknowledge the support of Miss. Afshan Khanum from Department of Biostatistics, FMH College of Medicine \& Dentistry, Lahore, Pakistan, for helping in data analysis on SPSS.

\section{CONFLICT OF INTEREST}

None to declare

\section{FINANCIAL DISCLOSURE}

None to disclose

\section{REFERENCES}

1. Bhatti UA, Qureshi B, Azam S. Trends in Contemporary Endodontic Practice of Pakistan: A National Survey. J Pak Dent Assoc. 2018;27:50-7.

https://doi.org/10.25301/JPDA.272.50

2. Raoof M, Heidaripour M, Shahravan A, Haghani J, Afkham A, Razifar M, et al. General dental practitioners' concept towards using radiography and apex-locators in endodontics. Iran Endod J. 2014;9:277.

3. Weiger R, Axmann-Krcmar D, Lm C. Prognosis of conventional root canal treatment reconsidered. Dent Traumatol. 1998;14: $1-9$.

https://doi.org/10.1111/j.1600-9657.1998.tb00801.x

4. Akhlaghi N, Nourbakhsh N, Khademi A, Karimi L. General dental practitioners' knowledge about the emergency management of dental trauma. Iran Endod J.2014;9:251.

5. Weisleder R, Yamauchi S, Caplan DJ, Trope M, Teixeira FB. The validity of pulp testing: a clinical study. J Am Dent Assoc.2009; 140:1013-7.

https://doi.org/10.14219/jada.archive.2009.0312

6. Kaufman AY. An enigmatic sinus tract origin. DENT TRAUMATOL. 1989;5:159-61.

https://doi.org/10.1111/j.1600-9657.1989.tb00353.x

7. Loest C. Quality guidelines for endodontic treatment: consensus report of the Eur Soci Endodontol. Int Endod J. 2006;39:921-30. https://doi.org/10.1111/j.1365-2591.2006.01180.x

8. Sjögren U, Hägglund B, Sundqvist G, Wing K. Factors affecting the long-term results of endodontic treatment. J Endod. 1990;16:498504.

https://doi.org/10.1016/S0099-2399(07)80180-4

9. Eriksen HM. Endodontology-epidemiologic considerations. DENT TRAUMATOL. 1991;7:189-95. https://doi.org/10.1111/j.1600-9657.1991.tb00434.x

10. Kohli A, Singh S, Podar R, Dadu S, Kulkarni G. A comparative evaluation of endodontic practice trends in India:" The Mumbai study". Indian J Dent Sci. 2014;25:729.

https://doi.org/10.4103/0970-9290.152179

11. Ghaffar A, Zaidi S, Qureshi H, Hafeez A. Medical education and research in Pakistan. Lancet. 2013;381(9885):2234-6. https://doi.org/10.1016/S0140-6736(13)60146-4

12. Jawaid SA. Plight of Dentistry in Pakistan. Pak J Med Sci Q. 2020;36:299.

https://doi.org/10.12669/pjms.36.3.2278

13. Palmer N, Ahmed M, Grieveson B. An investigation of current endodontic practice and training needs in primary care in the north west of England. Br Dent J. 2009;206(11):E22-E.

https://doi.org/10.1038/sj.bdj.2009.473

14. Unal GC, Kaya BU, Tac AG, Kececi AD. Survey of attitudes, materials and methods preferred in root canal therapy by general dental practice in Turkey: Part 1. Eur J Dent. 2012;6:376.

https://doi.org/10.1055/s-0039-1698975

15. Clarkson J, Ramsay C, Aceves M, Brazzelli M, Colloc T, Dave $\mathrm{M}$, et al. Recommendations for the re-opening of dental services: a rapid review of international sources. 2020.

16. Carr GB, Murgel CA. The use of the operating microscope in endodontics. Dent Clin N Am. 2010;54:191-214.

https://doi.org/10.1016/j.cden.2010.01.002

17. Darcey J, Taylor C, Roudsari RV, Jawad S, Hunter M. Modern endodontic principles part 3: preparation. Dent Update. 2015;42:81022.

https://doi.org/10.12968/denu.2015.42.9.810

18. Gordon M, Chandler N. Electronic apex locators. Int Endodo J. 2004;37:425-37.

https://doi.org/10.1111/j.1365-2591.2004.00835.x 
19. Lee M, Winkler J, Hartwell G, Stewart J, Caine R. Current trends in endodontic practice: emergency t reatments and technological armamentarium. J Endod. 2009;35:35-9.

https://doi.org/10.1016/j.joen.2008.10.007

20. Shantiaee Y, Dianat O, Mosayebi G, Namdari M, Tordik P. Effect of root canal preparation techniques on crack formation in root dentin. J Endod. 2019;45:447-52. https://doi.org/10.1016/j.joen.2018.12.018

21. Tomson PL, Simon SR. Contemporary cleaning and shaping of the root canal system. Prim Dent J. 2016;5:46-53.

22. Mittal R, Singla MG, Garg A, Dhawan A. A comparison of apical bacterial extrusion in manual, ProTaper rotary, and one shape rotary instrumentation techniques. J Endod. 2015;41:2040-4.

https://doi.org/10.1016/j.joen.2015.09.002

23. Christofzik D, Bartols A, Faheem MK, Schroeter D, GroessnerSchreiber B, Doerfer CE. Shaping ability of four root canal instrumentation systems in simulated 3D-printed root canal models. PLoS One. 2018;13(8):e0201129.

https://doi.org/10.1371/journal.pone.0201129

24. Darcey J, Jawad S, Taylor C, Roudsari RV, Hunter M. Modern endodontic principles part 4: irrigation. Dent Update. 2016;43:20-33. https://doi.org/10.12968/denu.2016.43.1.20

25. Neelakantan P, Ounsi HF, Devaraj S, Cheung GS, Grandini S. Effectiveness of irrigation strategies on the removal of the smear layer from root canal dentin. Odontology. 2019;107:142-9.

https://doi.org/10.1007/s10266-018-0373-2

26. Gaikwad A, Jain D, Rane P, Bhondwe S, Taur S, Doshi S. Attitude of general dental practitioners toward root canal treatment procedures in India. J Contemp Dent Pract. 2013;14:528-31.

https://doi.org/10.5005/jp-journals-10024-1356

27. Galler K, Grubmüller V, Schlichting R, Widbiller M, Eidt A, Schuller C, et al. Penetration depth of irrigants into root dentine after sonic, ultrasonic and photoacoustic activation. Int Endod J. 2019;52:1210-7.

https://doi.org/10.1111/iej.13108

28. Gluskin AH, Lai G, Peters CI, Peters OA. The double-edged sword of calcium hydroxide in endodontics: Precautions and preventive strategies for extrusion injuries into neurovascular anatomy. J Am Dent Assoc. 2020.

https://doi.org/10.1016/j.adaj.2020.01.026

29. Iqbal A, Akbar I, Qureshi B, Sghaireen MG, AL-Omiri MK. A survey of standard protocols for endodontic treatment in north of KSA. Int Sch Res Notices. 2014;2014.

https://doi.org/10.1155/2014/865780

30. Darcey J, Roudsari RV, Jawad S, Taylor C, Hunter M. Modern endodontic principles part 5: obturation. Dent Update. 2016;43:11429.

https://doi.org/10.12968/denu.2016.43.2.114 